\title{
A Rare Case of Post-Insect Bite Periocular Necrotising Fasciitis: Successful Surgical Management
}

\author{
Rajan Sharma ${ }^{1 *}$, Rani Sujatha ${ }^{1}$, Mahesh A ${ }^{2}$, Ashok Sharma ${ }^{3}$, K S Nagaraja ${ }^{1}$, and Aabid Maqbool
}

${ }^{1}$ Department of Ophthalmology, Dr. B. R. Ambedkar Medical College \& Hospital, Bengaluru, Karnataka, India

${ }^{2}$ Department of Plastic surgery, Dr. B. R. Ambedkar Medical College \& Hospital, Bengaluru, Karnataka, India

${ }^{*}$ Corresponding author: Sharma R, Junior Resident, Department of Ophthalmology, Dr. B. R. Ambedkar Medical College \& Hospital, Bengaluru, Karnataka, India, E-mail: rajansharma122@gmail.com.

Received date: Aug 02, 2017; Accepted date: Sep 06, 2017; Published date: Sep 08, 2017

Citation: Sharma R, Sujatha R, Mahesh A, Sharma A, Nagaraja KS, et al. (2017) A Rare Case of Post-Insect Bite Periocular Necrotising Fasciitis: Successful Surgical Management. J Eye Cataract Surg Vol.3 Iss.3:38.

Copyright: @ 2017 Sharma R, et al. This is an open-access article distributed under the terms of the Creative Commons Attribution License, which permits unrestricted use, distribution, and reproduction in any medium, provided the original author and source are credited.

\section{Abstract}

A 80-year-old female of moderate build who presented with complaints of pain, redness and severe swelling of the lids of left eye since 3 days following an insect bite. On examination she had severe edema of left eyelids extending to upper cheek area, blackish discoloration of lower periorbital skin. Slit lamp biomicroscopy did not reveal any abnormality in either of eyes. Microbiological investigations both examination of Gram stained smear and cultures did not show any microorganism. The patient was initially started on broad-spectrum intravenous antibiotics but did not show favourable response. Therefore, surgical debridement in two sittings followed by reconstruction with cheek rotation flap was performed. Patient responded favourably to surgical treatment. This case demonstrates that ophthalmologists should have high index of suspicion as necrotising fasciitis may occur following insect bite even in immuno-competent patients. Emergent surgical management coupled with parenteral broad spectrum antibiotics may limit ocular morbidity.

Keywords: Necrotising fasciitis; Insect bite; Surgical debridement; Fascio-cutaneous rotation flap

\section{Introduction}

Necrotising fasciitis (NF) is a severe infection with rapidly progressing inflammation and extensive necrosis of the skin, subcutaneous fat and fascia. According to causative pathogens, NF is divided into Type I (poly-microbial etiology) which mostly affects the immunocompromised and Type II (mono-microbial etiology) occurs in immunocompetent individuals. The condition often occurs following a minor trauma. We report a rare case of periocular necrotising fasciitis following an insect bite successfully treated with parenteral antibiotics, surgical debridement \& reconstruction of defect with facial flap. To our knowledge periocular NF following insect bite is an extremely rare entity and one such case has been reported in a patient diagnosed with chronic lymphocytic leukemia. Periocular NF is associated with significant mortality (12.5\%) [1]. We emphasise on the importance of urgent surgical intervention to avoid mortality in periocular NF.

\section{Case Report}

An 80-year old female of moderate build presented to outpatient department with complaints of pain, redness and severe swelling of the lids since 3 days (Figure 1). Patient specified that she experienced an insect bite (unknown identity) in dark on left lower eyelid prior to onset of symptoms. She was not a known diabetic or hypertensive. The patient was not on regular medical care for any other systemic disease.

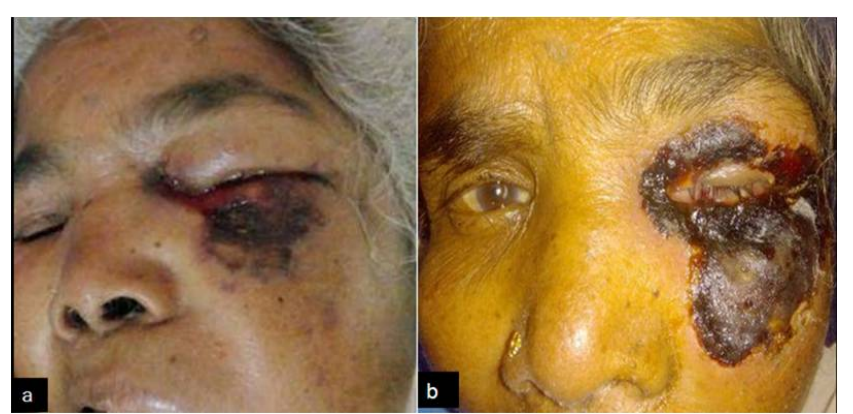

Figure 1: Photograph of the Left eye on (a) Presentation: widespread edema involving left eyelids extending to upper cheek area, with hyperaemia and crusting of lower perorbital skin; (b) 5 days after presentation: Necrosis of tissues involving left upper, lower eyelid and upper cheek

On examination she had widespread edema involving left eyelids extending to upper cheek area, with hyperaemia and crusting of lower perorbital skin. Slit lamp biomicroscopy did not reveal any abnormality in either of eyes.

On investigations, the total white blood cell count was 17,800 cells/mcL with neutrophilic leucocytosis. HBA1c reading was 6.3\%. Gram stain did not show any microorganism. Computerised axial tomography showed extensive left preseptal 
soft tissue thickening. Both eyeballs and extra ocular muscles were normal. Patient was immediately admitted and started on intravenous antibiotics including Amoxicillin/clavulunate potassium 1.2 g every $12 \mathrm{~h}$, Amikacin $500 \mathrm{mg}$ every $12 \mathrm{~h}$ and Metronidazole $400 \mathrm{mg}$ every $8 \mathrm{~h}$. She was also put on Moxifloxacin $0.5 \%$ eye drops TID and Moxifloxacin ointment at bed time with care of eyelids with antiseptic lotion. Over the next $72 \mathrm{~h}$, the periorbital swelling worsened and the patient was switched to Piperacillin+Tazobactam (tazotum) $4.5 \mathrm{~g}$ i.v BD and Amoxicillin/clavulunate was stopped. Necrosis of tissues started extending to left upper eyelid and upper cheek (Figure 2).

Surgical debridement was then performed in 2 separate sittings on days 5 and 13 (Figure 2a). Daily wound care including slough removal was performed (Figure 2b). Parenteral antibiotics were continued as before and nutrition supplementation was added with i.v fluids. Surface wound infection got controlled and healthy granulation tissue was observed at 6 weeks (Figure 2c). The final reconstruction was done with posterio-inferiorly based fascio-cutaneous cheek rotation flap on day 51 after initial presentation (Figures 2d-2f). Ectropion was present post-procedure but the patient showed reluctance to get correction done (Figure $2 \mathrm{~g}$ ).

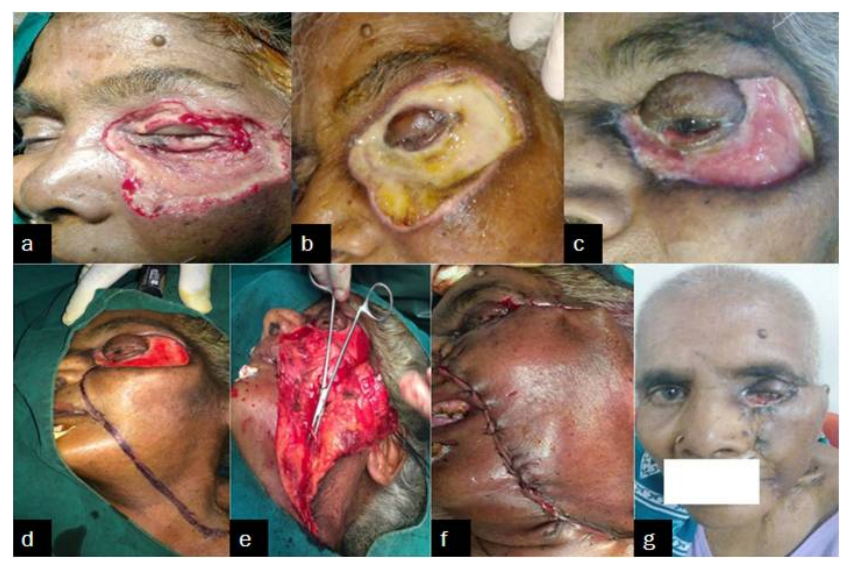

Figure 2: Photographs showing : (a)Large defect after first debridement and removal of necrotic tissue; (b)Sloughing in the defect with purulent discharge; (c)Healthy granulation tissue after regular slough removal; (d)Marking of flap before reconstruction (lower medial border of defect to nasolabial fold to inferior mandible margin extending to lower part of neck upto the clavicle); (e)Intraoperative photograph showing facial artery with perforators retained for blood supply to the flap; (f)Intraoperative photograph of flap inset; (g)Photograph 7 days post-reconstruction

\section{Discussion}

Necrotising Fasciitis (NF) is uncommon yet life threatening infection seen more commonly in immunocompromised or diabetic patients. The condition is most often caused by Streptococcus pyogenes or Staphylococcus aureus infection, Group A beta-hemolytic streptococci (NF Type II) being the most frequent bacterial etiology leading to acute and severe infection. Type-I NF is polymicrobial etiology and causative organisms include obligate and facultative anaerobes [1]. Nearly $10 \%$ of NF cases have been reported to involve the head and neck region [2]. Periorbital NF have been reported to have better prognosis [1]. Studies published in past few years suggest that the mortality rate in case of periorbital NF ranges from $8.5 \%$ [3] to $12.5 \%$ [1]. Wladis et al reported a case series of seventeen patients with periorbital necrotizing fasciitis. The authors observed that patients who did not have immunodeficiencies, had better visual outcome. However, patients with history of immunosuppression needed exenteration, but did not show significantly worse visual outcomes [4]. Our patient did not demonstrate any evidence of immune deficiency.

The antecedent condition in most cases is minor trauma [1]. In very rare cases an insect bite can be the inciting event. One such case of periocular NF following insect bite has been reported but the patient was immunocompromised being a diagnosed chronic lymphocytic leukemia [5]. Two other case reports were found, one involving forearm after mosquito bite and the second case following a tick bite on the arm which proved fatal despite adequate treatment $[6,7]$. We report a rare case of NF following insect bite involving periocular region in a immunocompetent patient.

Successful management of necrotising fasciitis requires early diagnosis and urgent surgical intervention. Wong $\mathrm{CH}$ et al in a retrospective study identified the delay of more than $24 \mathrm{~h}$ from admission to surgery as the single most important independent predictor of mortality in NF [8].

Early surgical debridement with removal of necrotic tissues and appropriate parenteral antibiotics are the mainstay of therapy. In our case reconstruction of the large defect was done with posterio-inferiorly based fascio-cutaneous cheek rotation flap after two sittings of debridement of necrotic tissue and regular removal of slough and purulent discharge. The surface infection got controlled, the wound got localized \& healthy granulation tissue appeared at 6 weeks. The final reconstruction was planned $\&$ the same was performed on 51st day after initial presentation

Our case demonstrates that ophthalmologists and physicians should have high index of suspicion as necrotising fasciitis may occur following insect bite and should be dealt with utmost seriousness as an emergency. Emergent surgical management coupled with parenteral broad spectrum antibiotics may limit ocular morbidity and mortality.

\section{References}

1. Kronish JW, McLeish WM (1991) Eyelid necrosis and periorbital necrotizing fasciitis: report of a case and review of literature. Ophthalmology 98: 92-98.

2. Childers BJ, Potyondy LD, Nachreiner R, Rogers FR, Childers ER, et al. (2002) Necrotising fasciitis: a fourteen-year retrospective study of 163 consecutive patients. Am Surg 68: 109-116.

3. Amrith S, Hosdurga Pai V, Ling WW (2013) Periorbital necrotising fasciitis-a review. Acta Ophthalmol 91: 596-603.

4. Edward J. Wladis, Flora Levin, Roman Shinder (2015) Clinical Parameters and Outcomes in Periorbital Necrotizing Fasciitis. Ophthal Plast Reconstr Surg 31: 467-469. 
5. Cornelia Poitelea, Michael J (2005) Wearne. Periocular Necrotising Fasciitis: A Case Report. Orbit 24: 215-217.

6. Verma SB (2003) Necrotizing fascitis induced by mosquito bite. J Eur Acad Dermatol Venereol 17: 591-593.

7. Fernando DM, Kaluarachchi $\mathrm{Cl}$, Ratnatunga CN (2013) Necrotizing fasciitis and death following an insect bite. Am J Forensic Med Pathol 34: 234-236
8. Wong CH, Chang HC, Pasupathy S, Khin LW, Tan JL, et al. (2003) Necrotizing fasciitis: clinical presentation, microbiology, and determinants of mortality. J Bone Joint Surg Am 85-A: 1454-1460. 